LETTER

\section{Association between time from SARS-CoV-2 onset to case confirmation and time to recovery across sociodemographic strata in Singapore}

Amid the coronavirus disease-2019 (COVID-19) pandemic, one of the most important indices of healthcare systems' performance in addressing the drastically increased burden is the average time to recovery of patients, the minimization of which indicates a strong capacity in handling the crisis and avoiding a total collapse of the systems. Previous research has suggested the importance of early detection amid epidemic outbreaks to facilitate better management of the disease. ${ }^{1}$ Nevertheless, seldom has any research examined the relationship between time from the onset of severe acute respiratory syndrome coronavirus 2 (SARS-CoV-2) to case confirmation and time to recovery, as well as how this relationship varies across sociodemographic strata.

From the Singaporean official website on COVID-19, ${ }^{2}$ I extracted the records of 221 recovered patients with symptomatic presentation in Singapore, where the mortality rate from SARS-CoV-2 was estimated at only $0.09 \%$ as of May 2020. ${ }^{3}$ Although a large fraction of the patient data was pending further update, the

Table 1 Relative risks (RR) of increased time to recovery from SARS-CoV-2 with 95\% Cls estimated from Poisson regression

\begin{tabular}{lll}
\hline & RR $(95 \% \mathrm{CI})$ & P value \\
\hline Local transmission (imported case as referent) & $0.85(0.78,0.93)$ & 0.000 \\
\hline Age (in 10 years) & $1.08(1.04,1.12)$ & 0.000 \\
\hline Male (female as referent) & $1.02(0.91,1.15)$ & 0.735 \\
\hline Nationality (Singaporean as referent) & & \\
\hline Western nationalities & $0.87(0.72,1.04)$ & 0.131 \\
\hline South East Asian & $0.84(0.68,1.04)$ & 0.118 \\
\hline Other nationalities & $1.00(0.76,1.30)$ & 0.983 \\
\hline Number of days from onset to confirmation & $0.50(0.40,0.63)$ & 0.000 \\
\hline Interaction terms & & \\
\hline \multicolumn{1}{|c|}{ Age $\times$ Number of days from onset to confirmation } & $1.05(1.01,1.09)$ & 0.016 \\
\hline Male $\times$ Number of days from onset to confirmation & $1.17(1.04,1.33)$ & 0.012 \\
\hline Western nationalities $\times$ Number of days from onset to confirmation & $1.00(0.77,1.31)$ & 1.000 \\
\hline South East Asian $\times$ Number of days from onset to confirmation & $1.69(1.24,2.29)$ & 0.001 \\
\hline Other nationalities $\times$ Number of days from onset to confirmation & $0.83(0.57,1.20)$ & 0.320 \\
\hline
\end{tabular}

SARS-CoV-2, severe acute respiratory syndrome coronavirus 2 . currently available data will suffice for preliminary purposes. Using this data, a Poisson regression analysis was implemented to examine the aforementioned relationship, with age, sex and nationality specified as potential moderators respectively interacting with time from onset to case confirmation in relation to time to recovery. As only secondary analysis of publicly available data was involved, no ethics approval was required.

Results showed that being 10-year older was associated with $8 \%$ more time to recovery and one additional week from onset to case confirmation was associated with a $50.0 \%$ less time to recovery among Singaporean female. This inverse association was $17 \%$ weaker among male, 5\% weaker being 10-year older and 69\% weaker with other South East Asian nationalities. Full numeric results are tabulated as table 1 .

The observed inverse relationship between time from onset to case confirmation and time to recovery is possibly due to a lower severity of the condition among patients with only mild symptoms, which took longer to arouse medical attention but eventually less time to treat. The increased complexities among male and older patients suggested in previous research $^{4}$ may explain the observed weaker negative association, because these patients may be more likely to develop severe symptoms regardless of the time from onset to case confirmation. Last, the weaker association among South East Asian patients was possibly because of the systematic testing for foreign workers

\section{Accepted 16 May 2020}

J Epidemiol Community Health 2020;74:678.

doi:10.1136/jech-2020-214516

\section{ORCID iD}

Francisco Tsz Tsun Lai http://orcid.org/0000-00029121-1959

\section{REFERENCES}

1 Volchkov V, Titova O, Larin D, et al. Intensive care of patients with severe influenza during the epidemic in 2016. Eur Respir J 2017:50:PA2125.

2 Singaporean Government. COVID-19 Singapore Dashboard. 2020. Available https://co.vid19.sg/singa pore/ (accessed 6 May 2020)

3 Varley K. As virus deaths grow, two rich nations keep fatality below $0.1 \%$. Bloomberg, 2020. Available https://www.bloomberg.com/news/articles/2020-0505/as-virus-deaths-grow-two-small-nations-keepfatality-below-0-1

4 Li X, Xu S, Yu M, et al. Risk factors for severity and mortality in adult COVID-19 inpatients in Wuhan. J Allergy Clin Immunol 2020.

5 Tan Y, Illmer A. Coronavirus: Singapore spike reveals scale of migrant worker /infections. BBC News, 2020 Available https://www.bbc.com/news/world-asia $-52320289$ 